\title{
LII. On electro-magnetism
}

\section{Mr.J. Tatum}

To cite this article: Mr. J. Tatum (1823) LII. On electro-magnetism, Philosophical Magazine Series 1, 61:300, 241-243, DOI: 10.1080/14786442308644312

To link to this article: http://dx.doi.org/10.1080/14786442308644312

$$
\text { 曲 Published online: } 27 \text { Jul } 2009 .
$$

Submit your article to this journal 전

LII Article views: 3

Q View related articles $\asymp$ 


\title{
PHILOSOPHICAL MAGAZINE AND JOURNAL.
}

\author{
$30^{\mathrm{th}} A P R I L 1823$.
}

LII. On Electro-Magnetism. By Mr. J. Ta'vum.

To the Editors of the Philosophical Magazine and Jounal.

\author{
Genthemen,
}

QINCE the discovery of electro-magnetism by Professor $\omega$ CErsted*, much new and valuable information has been given to the scientific world by the united labours of various philosophical gentlemen on this very interesting subject. But from the apparent anomalies attending some experiments, a difference of opinion has been advanced by persons possessing great depth of penetration as well as considerable experimental knowledge: for it must be acknowledged that, from some experiments, it appeared as though the wire connecting the zinc and copper plates of a Voltaic apparatus possessed dissimilar poles on its opposite sides. But in order to be satisfied respecting the accuracy of this appearance, I contrived the apparatus so that I could carry the needle all round the wire when it was in a perpendicular situation; or, the roire round the needle; or, I could rotate the wire as on an axis, so as to present every side of it to the same part of the needle: by which means I found that the same part of the needle was invariably attracted or repelled; so that the whole periphery of the wire appears to possess the same polarity. But as the construction of the common needle does not allow it to rotate in a vertical direction, I could not so satisfactorily investigate the properties of an horizontal wire. I therefore removed the agate cap of a compass, and suspended it by an horizontal axis, so that it could rotate freely in a vertical direction; and by this means I was enabled to examine the magnetic state of the circumference of this wire in a more satisfactory manner, which proved to be of an uniform polarity.

In the early part of the above investigation, and about the time Mr. Faraday's paper on this subject appeared, in which he stated that he had discovered the needle possessed eight poles, -namely, two north and two south, on each side of the needle's equator, -I thought I had observed sixteen poles; namely, * Phil. Mag. vol. lvii. pp. 40, 203, 257, \&c.

Vol.61. No. 300. April 1823. H h 
four north and four south, on each side of the equator of the needle: and the above arrangement of my apparatus enabled me to investigate the subject more accurately than I formerly could, the results of which I now submit to the perusal of the readers of your Magazine.

But first I would premise, that when I speak of attraction or repulsion, as applicable to the perpendicular or horizontal wire, I do not mean a deviation of only three or four degrees, but that of a whole revolution.

Exp. 1.-Let A B (Plate IV. fig. 1,) represent part of the perpendicular wire connected with a Voltaic apparatus (hereafter to be described), and C D the position of one end of a magnetic needle to the wire. In such a position the needle will be attracted.

Exp, 2.-But if it be carried to the opposite side of the wire, and consequently present its opposite edge to it (as in fig. 2,) it will be repelled. Of course, here are two contrary poles in a transverse direction.

Exp. 3. - Let the equator of the needle be advanced a little nearer to the wire, as is represented in fig. 3 , but presenting the same edge of it to the wire as in fig. 1 ; in this position.it will be repelled. Of course, here is a dissimilar polarity exhibited to what there was in the first position.

Exp. 4.-When the needle is removed to the other side of the wire, as in fig. 4, consequently presenting its opposite edge to the wire, it will in this situation be attracted. Here again are two more contrary poles in a transverse direction, which with the two already noticed make four poles.

Exp. 5.-Let C D, fig. 5, represent an horizontal wire connected-with the apparatus; and E F, the under side of part of a needle (now mounted on an horizontal axis) above it. It will when so situated be attracted.

Exp. 6. - If its upper side be brought under the wire, as in fig. 6 , it will be repelled. Consequently, here are two dissimilar poles in a perpendicular direction, which with the four transverse ones already noticed make six; and as the extremity of the needle is possessed of two transverse and two perpendicular poles, it may be represented by N N S S, fig. 11 .

Exp. 7.- If the needle be advanced a little further, but above the wire, as in fig. 7, (of course presenting the same side to the wire as in fig. 5 , ) it will there be repelled.

Exp. 8. - If the needle be removed to below the wire, but with its point at the same distance from it, as in fig. 8 , it will be attracted.

Here again are two more dissimilar poles in a perpendicular direction; so that there are four transverse and four perpendicular 
Fig. 1.

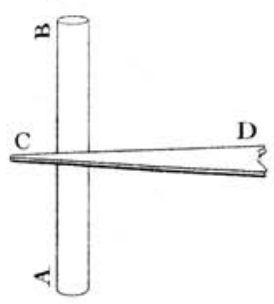

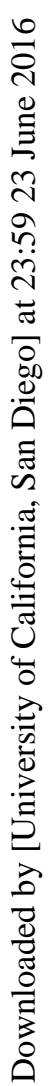
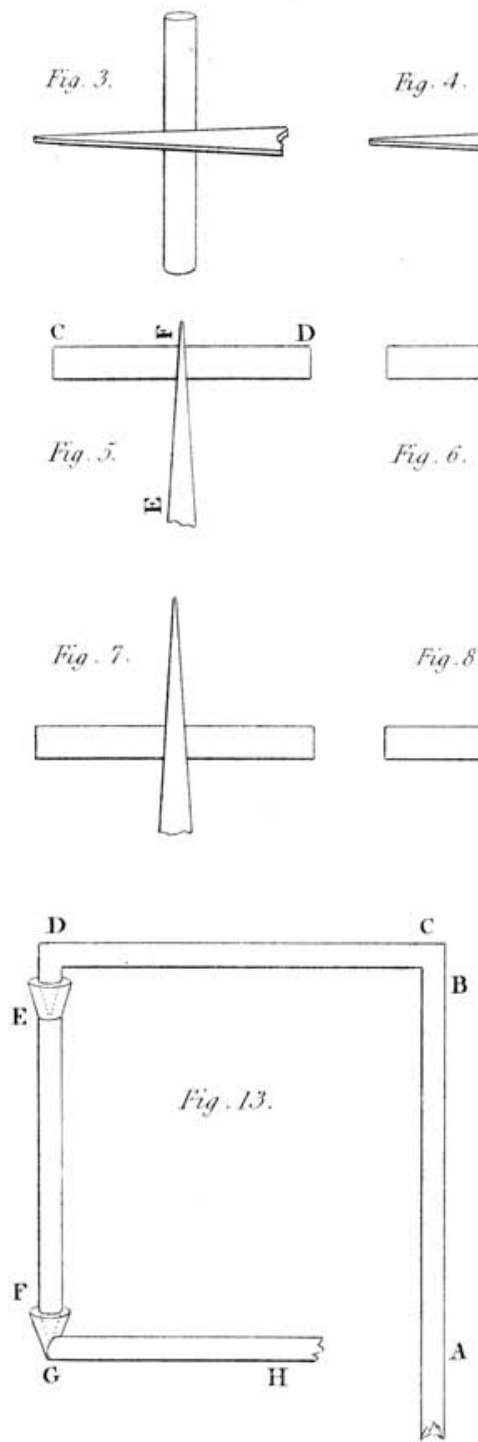

Fig. 9 Fig. 10 ,
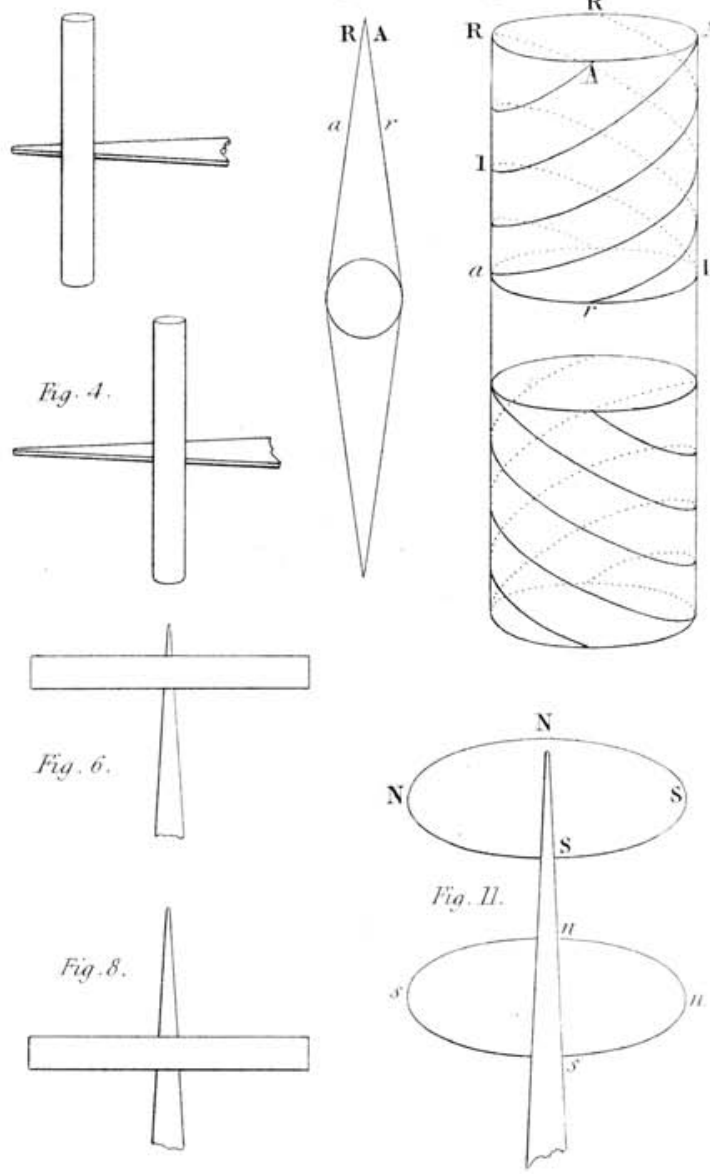

Fig. 72.

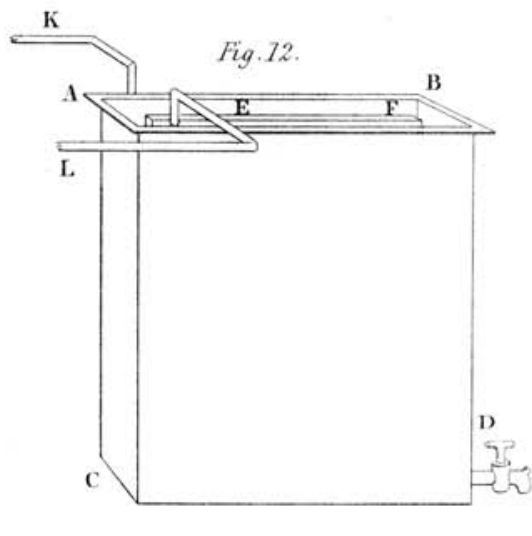


pendicular poles, making eight poles on one side of the equator of the needle, which may be represented by N N S S and $n n s s$, fig. 11.

And as similar effects are produced on the opposite side of the equator of the needle (but with this difference, that attraction is produced instead of repulsion, and vice versâ), the needle must possess at least sixteen poles.

But if the results of the experiments related at the commencement of this paper be not borne in mind, it might be thought that these attractions and repulsions resulted from dissimilar poles on opposite sides of the connecting wire :- but this I think I have proved is not the case.

Perhaps these phænomena may be further illustrated by R A ar, fig. 9, which may represent the two edges of a mag. netic needle with its transverse poles, in which $R$ on the left hand may represent a north pole, and $A$ on the right hand a south pole: and also $a$ on the left a south pole, and $r$ on the right a north pole. But I do not consider that the magnetic fluid circulates in straight lines in a transverse direction, as from $\mathrm{R}$ to $\mathrm{A}$, or in a perpendicular direction, as from $\mathrm{R}$ to $a$; but that it circulates in a spiral direction, making one complete revolution, as from $A 1$ to $R$ in the upper part of fig. 10; the letters of which figure will represent the eight transverse and perpendicular poles; and the several spiral lines, the direction of the fluid from and to the four opposite poles; and the lower part of the same figure may represent the opposite end of the needle, where the spirals indicate the fluid as circulating in an opposite direction.

This spiral direction of the fluid will, I consider, account for the motion of that excellent little rotatory cylindrical apparatus of M. Ampere, which it is well known rotates in an opposite direction when placed on a dissimilar pole of the magnet. It will also, in my opinion, account for the advancing motion of the closed helix of M. de la Rive up the magnet, as well as the rotation of a magnet round a wire, and a wire. round a magnet, invented by Mir. Faraday.

And as I consider that the fluid from the copper side of the Voltaic apparatus circulates in an opposite spiral direction to what it does when passing from the zinc side, I thought that if I varied M. Ampere's apparatus, by substituting zinc in the place of his double cylinder of copper, and copper in the place of his single cylinder of zinc, it would produce a contrary rotation;-which fully answered my expectation.

There also appears a coincidence in the spiral direction of the fluid, and the helixes when forming part of the Voltaic circuit; for when pieces of steel are placed in a left-handed $\mathrm{Hh} 2$ helix 
helix to be magnetized, their poles will be in an opposite direction to what would have been produced by a right-handed one.

I think this contrary spiracular circulation of the fluid, when proceeding from opposite poles of a Voltaic apparatus; will account for the attraction of parallel wires when proceeding from Voltaic poles similarly situated; but repelled when proceeding from poles dissimilarly situated, for then the circulation of the fluids round the wires will be opposite to each other, and of course will repel. This may be illustrated by placing a left- and right-handed helix parallel to each other ; for so situated, the direction of these coils will be seen to be opposite to each other; which will represent the course of the fluid round parallel wires proceeding from poles oppositely situated.

The apparatus which $I$ made use of to produce the effects on the needle consists of a copper vessel A B C D, fig. 12, two feet deep, upwards of twenty-two inches broad, and nearly two inches and a half wide, in which is placed a plate of zine E F, twenty-three inches deep, and full twenty inches broad, with a wire L proceeding from the zinc, and another wire $\mathrm{K}$ attached to the copper, on which I can slip horizontal, perpendicular, and helix wires. The vessel holds about six gallons of diluted acid, which added to the weight of the zinc plate (a quarter of a cwt.) renders it too heavy to decant the fluid. It is therefore provided with a cock, by which the diluted acid can be drawn off without any labour. When this apparatus was fresh charged with nitro-sulphuric acid twelve times diluted, its magnetic properties were so powerful, that it influenced a large heavy needle about two feet distant; it also rendered pieces of steel magnetic, which were placed in a helix sufficiently strong to lift each other, before the vessel could be quite filled with the diluted acid, although it was poured as quick as possible from a four-gallon carboy. Its attracting and repelling properties were much too strong to make the above experiments, until the acid had remained in the apparatus some time, by which its power was reduced. This apparatus, when in full action, is admirably calculated to show the dissimilar effects of right- and left-handed helixes-on pieces of steel to be magnetized.

Dorset-street, April 9, 1823.

I am yours, \&c.

About a week ago I sent you a paper on Electro-magnetism, in which I noticed, that the magnetic fluid circulated round the north end of a magnet in a different direction to what it did round the south end. This opinion was in consequence 
of the dissimilar effects produced on the opposite ends of the needle when applied to the electro-magnetic apparatus. I also observed, that I considered a similar difference was produced by the wire connected with the copper end of a Voltaic apparatus, to what was produced by that which was united with the zinc end. I have since thought it might not be amiss to introduce a simple figure of the apparatus by which I ascertained these opposite effects.

Let ABCDEFGH (fig. 13) represent the wire connecting the two poles of the apparatus; the part $A$ connected with the copper, and the part $\mathrm{H}$ with the zinc:- this is not one continued wire, for I wished to have the opportunity of rotating the perpendicular part $\mathrm{EF}$, which is represented with a small cup at $E$, and terminating in a point $F$, which dips into another small cup, both of which are filled with mercury, which forms a complete connexion. While the apparatus is thus situated, it will be found that the very opposite effects are produced from $\mathrm{HGF}$ to $\mathrm{E}$, to what are produced from $\mathrm{A} \mathrm{B} \mathrm{C}$ to D: as for instance, if one end of a needle be brought under the horizontal part of the wire $\mathrm{CD}$, it will be attracted; but if brought under the horizontal part $\mathrm{GH}$, it will be repelled : and if brought to the right side of the perpendicular part $A B$, it will be attracted; but if removed to the right side of the perpendicular part $\mathrm{EF}$, it will be repelled. If the equator needle be carried nearer to the wire, the reverse of the above effects is produced, as was shown in my former paper. From which I deduce, that a contrary circulation of the fluid is produced by the positive side of the apparatus, to what is exhibited by the negative side.

I scarcely need observe, that these effects will be applied by every one to the rotatory copper and zinc cylinders of Ampere, in which it is well known that the copper rotates in an opposite direction to that of the zinc.

J. T.

P.S.-I hope in the course of a few weeks to have a little more leisure, when perhaps I shall be enabled to resume this interesting subject.

LIII. True apparent Right Ascension of Dr. MaSk ELYNE's 36 Stars for every Day in the Year 1823, at the Time of passing the Meridian of Greenroich.

[Continued from page 199.]

N.B. On those days where an Asterisk is prefixed the Star passes twice; the $R$ given is that at the first passage. 